\title{
Enquête
}

Archives de la revue Enquête

2| 1995

Usages de la tradition

\section{Inventer des traditions}

Inventing Traditions

\section{Eric Hobsbawm}

Traducteur : André Mary, Karim Fghoul et Jean Boutier

\section{CpenEdition}

Journals

\section{Édition électronique}

URL : http://journals.openedition.org/enquete/319

DOI : 10.4000/enquete.319

ISSN : 1953-809X

Éditeur :

Cercom, Éditions Parenthèses

Édition imprimée

Date de publication : 31 décembre 1995

Pagination : 171-189

\section{Référence électronique}

Eric Hobsbawm, «Inventer des traditions », Enquête [En ligne], 2 | 1995, mis en ligne le 10 juillet 2013, consulté le 30 avril 2019. URL : http://journals.openedition.org/enquete/319; DOI : 10.4000/ enquete.319 


\title{
Inventer des traditions
}

\author{
Inventing Traditions
}

\author{
Eric Hobsbawm
}

Traduction : André Mary, Karim Fghoul et Jean Boutier

1 Eric Hobsbawm est professeur d'histoire au Birkbeck College, l'une des rares institutions d'enseignement pour adultes de l'université de Londres, lorsqu'il rédige cette introduction à l'ouvrage collectif désormais célèbre, The Invention of Tradition, issu d'un colloque organisé par la revue Past and Present ${ }^{1}$. Né en 1917 à Alexandrie, de mère autrichienne et de père anglais, il étudie à Vienne, Berlin, Londres, puis Cambridge, où il enseigne à King's College, de 1949 à 1955. Membre dès 1946 du Groupe des Historiens du parti communiste anglais, il est l'un des fondateurs, en 1952, de la revue Past and Present qui, dans les années soixante, est un des lieux d'échanges actifs entre l'histoire et les autres sciences sociales comme l'anthropologie et la sociologie. Son ceuvre est consacrée à l'histoire sociale et économique des mondes modernes et contemporains, dont il a proposé une lecture originale dans une série d'ouvrages, depuis L'Ère des révolutions (1962), L'Ère du capital (1975), L'Ère des empires (1990), jusqu'au Age of Extremes (1995), inédit en français. L'un des principaux protagonistes, dans l'après-guerre, de la constitution en Grande-Bretagne d'une histoire sociale qui se veut une "histoire des sociétés", il s'attache aux groupes dominés ou déviants, et développe, avec George Rude et quelques autres, une " histoire vue d'en bas ». Parmi d'autres travaux consacrés aux mouvements sociaux ou aux révoltés, signalons un ouvrage traduit en français, Nations et nationalismes (1990), particulièrement éclairant par rapport aux exemples évoqués dans ce texte et au questionnement concernant la résurgence contemporaine des nationalismes ethniques.

2 «Inventer la tradition»: l'effet d'annonce, qui consiste à laisser entendre qu'une tradition communautaire que l'on pensait immémoriale n'est en fait qu'une création récente liée aux enjeux d'un passé proche et aux stratégies de tel ou tel groupe social, est loin d'être épuisé. Comme le soulignent la présence de l'africaniste T. Ranger, mais aussi la participation de l'anthropologue B. S. Cohn, le paradigme initial des "traditions inventées" est né entre autres d'une rencontre entre l'histoire et l'anthropologie, d'où le double mouvement d'historicisation d'un présent ethnographique ou folklorique trop rapidement confondu avec une réalité ancestrale et 
d'anthropologisation du regard historique qui conduit notamment à insister sur la dimension rituelle et symbolique des pratiques « traditionnelles».

La proposition a suscité un vaste débat qui a particulièrement mobilisé les anthropologues et, plus récemment, tous ceux qui s'attachent à l'étude des phénomènes nationaux². S'il a ainsi conduit certains des co-auteurs à préciser, ou à nuancer leur propos ${ }^{3}$, il ne faudrait pas pour autant négliger cette formule paradoxale qui a largement dépassé le contexte britannique, où elle faisait figure d'une provocation. Son succès peut se mesurer à l'inflation des titres qui en ont repris sinon l'esprit du moins les termes. Il a toutefois quelque peu gommé le fait que les «traditions inventées", qu'Hobsbawm distingue des traditions anciennes véritables, sont pour l'essentiel des productions de la modernité visant à réintroduire des lieux de référence, des repères stables dans un monde soumis au changement et confronté à un certain vide social. L'inventivité de ce néotraditionalisme, particulièrement illustré par les productions du folklore nationaliste $\mathrm{du} X I X^{e}$ siècle, consacre en réalité le déclin de la tradition authentique et la mort de la coutume. D'où cette inversion singulière qui conduit Hobsbawm à identifier, dans ce contexte, la tradition au formalisme du rituel et de l'apparat, au souci de la répétition et de l'invariance, par contraste avec la réalité substantielle d'un ordre de la coutume à la fois fidèle à lui-même et néanmoins flexible et adaptable.

On pourrait penser que ce clivage typique de la modernité introduit à une simple redéfinition des frontières entre une anthropologie de la coutume ou des traditions authentiques et une histoire des traditions inventées, mais la contribution de T. Ranger élargit la portée du paradigme initial et la problématique de l'usage des traditions. L'hypothèse d'une invention proprement coloniale des traditions africaines, ou plus largement exotiques, conforme à l'idée que l'Europe de la fin du XIXe se faisait de la tradition, a pour contrepartie l'attention portée au mouvement d'appropriation des néo-traditions européennes par les populations indigènes. Le paradoxe atteint ici un sommet puisque les néo-traditions européennes, militaires, religieuses ou sportives, se révèlent être les vecteurs de la modernisation de l'Afrique. Bousculant les lieux communs de la rhétorique de la tradition et de la modernité, le paradigme de la tradition inventée permet ainsi d'appréhender une gamme très large de phénomènes de réinterprétation symbolique qui montrent que la représentation d'une légitimité intrinsèque $d u$ «traditionnel» est en fait sans cesse réinstrumentalisée, et cela au sein même des sociétés que l'on dit traditionnelles.

ANDRÉ MARY, KARIM FGHOUL, JEAN BOUTIER

Rien ne parait plus ancien et plus lié à un passé immémorial que l'apparat dont s'entoure la monarchie britannique dans ses cérémonies publiques. Or, comme l'établit un chapitre de ce livre ${ }^{4}$, cet apparat est, dans sa forme moderne, un produit de la fin $\mathrm{du} \mathrm{XIx}^{\mathrm{e}}$ et du $\mathrm{xx}^{\mathrm{e}}$ siècle. Des «traditions» qui semblent anciennes ou se proclament comme telles, ont souvent une origine très récente et sont parfois inventées. Quiconque est familier des collèges des anciennes universités britanniques sera en mesure de penser l'institution de telles «traditions » à une échelle locale, même si certaines d'entre elles - comme la fête annuelle des Nine lessons and carols dans la chapelle de King's College à Cambridge la veille de Noël - ont pu être banalisées par le moyen moderne de communication de masse qu'est la radio. Cette observation a constitué le point de départ d'un colloque organisé par la revue historique Past and Présent, base du présent ouvrage.

6 L'expression «tradition inventée » est utilisée dans un sens large, mais non pour autant imprécis. Elle inclut à la fois les «traditions » qui ont été effectivement inventées, 
construites et instituées de manière très officielle, et celles qui émergent de façon plus indistincte au cours d'une période brève et datable - peut-être quelques années à peine et s'établissent d'elles-mêmes avec une grande rapidité. En Grande-Bretagne, les vœux royaux de Noël à la radio (institués en 1932) répondent au premier cas de figure; l'apparition et le développement des pratiques associées à la finale de la Coupe dans la fédération britannique de football relèvent du second. Il est évident que toutes ne sont pas également permanentes, mais c'est leur apparition et leur établissement, plutôt que leurs chances de survie, qui constituent notre préoccupation première.

7 Les «traditions inventées» désignent un ensemble de pratiques de nature rituelle et symbolique qui sont normalement gouvernées par des règles ouvertement ou tacitement acceptées et qui cherchent à inculquer certaines valeurs et normes de comportement par la répétition, ce qui implique automatiquement une continuité avec le passé. En fait, là où c'est possible, elles tentent normalement d'établir une continuité avec un passé historique approprié. Un exemple saisissant est le choix délibéré du style gothique pour reconstruire au XIX ${ }^{e}$ siècle le parlement britannique, et la même décision, tout aussi délibérée, après la Seconde Guerre mondiale, de le rebâtir exactement sur les mêmes plans qu'auparavant. Il n'est pas nécessaire que les temps historiques dans lesquels s'insère la nouvelle tradition soient lointains, ni de remonter jusqu'à la nuit des temps. Les révolutions et les «mouvements progressistes" qui rompent avec le passé ont, par définition, leur propre rapport au passé bien qu'il puisse être coupé à une date précise, telle que 1789. Toutefois, même lorsqu'il existe une telle référence à un passé historique, la particularité des traditions « inventées » tient au fait que leur continuité avec ce passé est largement fictive. En bref, ce sont des réponses à de nouvelles situations qui prennent la forme d'une référence à d'anciennes situations, ou qui construisent leur propre passé par une répétition quasi obligatoire. C'est le contraste entre le changement permanent, l'innovation du monde moderne et la tentative de structurer au moins certaines parties de la vie sociale comme immuables et invariantes, qui rend «l'invention de la tradition " si intéressante pour les historiens des deux derniers siècles.

La «tradition », en ce sens, doit être clairement distinguée de la " coutume » qui domine les sociétés dites "traditionnelles ». L'objet et la caractéristique des "traditions», y compris des traditions inventées, c'est l'invariabilité. Le passé, réel ou fictif, auquel elles se réfèrent, implique des pratiques stables, formalisées de manière normative, se prêtant à la répétition. Dans les sociétés traditionnelles, la " coutume » a la double fonction du moteur et du volant. Elle n'exclut pas, jusqu'à un certain point, l'innovation et le changement, quoique de toute évidence l'exigence d'un changement qui soit en apparence compatible avec ce qui le précède, voire identique à lui, lui impose des limites substantielles. Elle donne donc à tout changement désiré (ou à toute résistance à l'innovation) la sanction d'un précédent, d'une continuité sociale et d'une loi naturelle telle qu'elle s'exprime dans l'histoire. Ceux qui étudient les mouvements paysans savent que la revendication d'un village concernant une terre « communale » ou un droit fondé sur la " coutume depuis des temps immémoriaux », traduit souvent non pas une réalité historique, mais l'équilibre des forces dans la lutte incessante du village contre les seigneurs ou contre d'autres villages. Ceux qui étudient le mouvement ouvrier britannique savent que la « coutume du métier » ou de l'échoppe peut ne pas représenter une tradition ancienne, mais n'importe quel droit que les travailleurs ont établi en pratique, parfois même récemment, et qu'ils espèrent maintenant étendre ou défendre en lui donnant la consécration de la perpétuité. La « coutume » ne peut se permettre d'être 
invariable, car même dans les sociétés "traditionnelles ", la vie ne l'est pas. Le droit coutumier ou la loi commune montre encore cette combinaison de flexibilité dans le contenu et d'attachement formel à ce qui précède. Cette différence entre «tradition » et " coutume », au sens où nous l'entendons, est ici bien illustrée. La " coutume » est ce que font les juges; la «tradition » (ici la tradition inventée) est la perruque, la robe et les autres attirails formels, les pratiques ritualisées qui entourent la substance de leur action. Le déclin de la « coutume » change inévitablement la «tradition» dans laquelle elle est habituellement imbriquée.

Une seconde distinction, moins importante, doit être faite entre la «tradition ", telle que nous l'entendons, et la convention ou la routine qui n'a pas de fonction symbolique ou rituelle significative en tant que telle, même si elle peut en acquérir une accessoirement. Il est évident que toute pratique sociale qui a besoin d'être effectuée de façon répétitive, va tendre, par commodité et efficacité, à développer un ensemble de telles conventions et routines, lesquelles vont être, de facto ou de jure, formalisées dans l'intention de faire connaître la pratique en question aux nouveaux pratiquants. Ceci s'applique aussi bien à des pratiques sans antécédent (comme le travail d'un pilote d'avion), qu'à celles qui sont depuis longtemps familières. Depuis la révolution industrielle, les sociétés ont été naturellement obligées d'inventer, d'instituer ou de développer, plus fréquemment que les sociétés antérieures, de nouveaux réseaux de conventions ou de routines de ce genre. Dans la mesure où elles fonctionnent mieux lorsqu'elles sont transformées en routines, en procédures automatiques ou même en actions réflexes, elles ont besoin d'invariance, ce qui peut gêner l'autre exigence, nécessaire, de la pratique : la capacité de faire face à des éventualités imprévues ou inhabituelles. Ceci est une faiblesse bien connue de la routinisation ou de la bureaucratisation, particulièrement au niveau subalterne où l'action invariable est généralement considérée comme la plus efficace.

De tels réseaux de conventions et de routines ne sont pas des « traditions inventées » tant que leurs fonctions, et donc leurs justifications, restent techniques plutôt qu'idéologiques (en termes marxistes, ils appartiennent à la « base » plutôt qu'à la « superstructure »). Ils sont conçus pour faciliter des opérations pratiques aisément identifiables; ils sont facilement modifiés ou abandonnés pour s'accorder à des besoins pratiques qui changent, en tenant toujours compte de l'inertie que toute pratique acquiert avec le temps ainsi que de la résistance émotionnelle à toute innovation des gens qui lui sont attachés. Il en est de même des « règles » reconnues des jeux ou d'autres modèles d'interaction sociale, là où elles existent, ou de toute norme établie de façon pragmatique. Lorsque ces règles sont combinées avec la "tradition ", la différence est facilement observable. Porter une bombe en montant à cheval a un sens pratique, comme le fait de porter un casque pour les motocyclistes ou les soldats; porter un type particulier de bombe avec l'habit rouge de la chasse à courre revêt un sens complètement différent. S'il n'en était pas ainsi, il serait aussi facile de changer le costume «traditionnel» des chasseurs de renard que d'introduire un casque de forme différente dans les armées - qui sont des institutions plutôt conservatrices - si l'on pouvait démontrer qu'il assure une protection plus efficace. En effet, nous pouvons supposer que les «traditions » et les conventions pratiques, ou routines, varient en fonction inverse. La « tradition » montre sa faiblesse lorsque, comme chez les juifs libéraux, les prohibitions alimentaires sont justifiées de manière pragmatique, par l'argument selon lequel les anciens Hébreux interdisaient le porc pour des raisons d'hygiène. Inversement, les objets ou les pratiques deviennent disponibles pour un plein usage symbolique et rituel quand ils ne sont plus entravés par leur 
utilisation pratique. L'éperon de l'uniforme des officiers de cavalerie est plus important pour la tradition quand il n'y a plus de cheval ; les parapluies des officiers de la garde en tenue civile perdent leur signification quand ils ne sont pas portés fermés (c'est-à-dire inutiles); les perruques des hommes de lois peuvent difficilement acquérir leur signification moderne tant que les autres individus ne cessent d'en porter.

Ce qui est assumé ici, c'est que l'invention des traditions est essentiellement un processus de formalisation et de ritualisation caractérisé par la référence au passé, ne serait-ce que par le biais d'une répétition imposée. Le processus réel de création de tels complexes rituels et symboliques n'a pas été étudié convenablement par les historiens. Une bonne partie demeure mal connue. L'exemple est sans doute plus clair quand une "tradition " est délibérément inventée et construite par un innovateur singulier, comme les boyscouts de Baden Powell. Peut-être est-ce presque aussi facile à suivre dans le cas de cérémonies instituées et planifiées officiellement dans la mesure où il $\mathrm{y}$ a des chances qu'elles soient bien documentées, comme dans l'exemple de l'élaboration du symbolisme nazi et des rassemblements du parti à Nuremberg. Il est probablement plus difficile de suivre ces processus-là où de telles traditions ont été partiellement inventées, et en partie développées dans des groupes privés (où il est moins probable qu'ils soient bureaucratiquement enregistrés), ou de façon informelle après une certaine période comme au parlement et dans les professions juridiques. La difficulté ne réside pas seulement dans les sources, mais aussi dans les techniques, quoique nous disposions, pour l'étude de tels objets, aussi bien de disciplines ésotériques spécialisées dans le symbolisme et le rituel, comme l'héraldique ou l'étude de la liturgie, que des méthodes historiques d'A. Warburg. Malheureusement, ces disciplines ne sont pas habituellement familières aux historiens de l'ère industrielle.

12 Aucun des moments ou des lieux qui intéressent les historiens, n'est probablement étranger à l'« invention » de la tradition comprise en ce sens. Nous pouvons toutefois supposer qu'elle est plus fréquente quand une transformation rapide de la société affaiblit ou détruit les modèles sociaux pour lesquels les anciennes traditions avaient été élaborées, et produit de nouveaux modèles auxquels elles ne peuvent plus s'appliquer; cela peut également se produire quand de telles traditions " anciennes», leurs supports et leurs diffuseurs institutionnels ne se montrent plus suffisamment adaptables et flexibles, ou sont éliminés; en bref, quand il y a des changements suffisamment vastes et rapides de l'offre ou de la demande. De tels changements ont été particulièrement significatifs dans les deux cents dernières années et il est donc raisonnable de penser que de telles formalisations instantanées de nouvelles traditions sont regroupées dans cette période. Ceci implique, entre parenthèses, à l'encontre aussi bien du libéralisme du XIX siècle, et plus récemment de la théorie de la "modernisation", que de telles formalisations ne sont pas limitées aux sociétés dites « traditionnelles», mais qu'elles ont aussi leur place, sous une forme ou une autre, dans les sociétés «modernes ». Il en est globalement ainsi, mais nous devons prendre garde d'avancer les suppositions suivantes : premièrement, que les formes les plus anciennes de structure communautaire et d'autorité, et par conséquent les traditions qui leur sont associées, sont inadaptables et deviennent très vite non viables, et deuxièmement, que les "nouvelles" traditions résulteraient tout simplement de l'impossibilité d'utiliser ou d'adapter les anciennes.

L'adaptation apparaît lorsque d'anciens usages sont confrontés à de nouvelles conditions et que de vieux modèles sont utilisés dans de nouveaux buts. Les anciennes institutions avec leurs fonctions établies, leurs références au passé, leurs langages et leurs pratiques 
rituelles sont parfois dans la nécessité de s'adapter de cette manière : l'Église catholique face à de nouveaux défis politiques et idéologiques et à des changements importants dans la composition des fidèles (telle la féminisation notable à la fois de la piété profane et du personnel clérical) ${ }^{5}$; les armées de métier confrontées à la conscription; d'anciennes institutions, comme les tribunaux, qui opèrent actuellement dans un contexte modifié, et parfois, avec des fonctions modifiées, dans de nouveaux contextes. Il en a été de même d'institutions bénéficiant d'une continuité nominale, mais qui se sont en fait transformées en quelque chose de très différent, comme les universités. Ainsi Bahnson ${ }^{6} \mathrm{a}$ analysé le déclin soudain, après 1848 , de la pratique traditionnelle de l'exode massif des étudiants des universités allemandes (à cause de conflits ou de manifestations), déclin qui est en relation avec les changements du prestige académique des universités, l'augmentation de l'âge de la population étudiante, son embourgeoisement qui réduit les tensions citadins/étudiants et le caractère séditieux des étudiants, la nouvelle institution de la libre circulation entre les universités, les changements qui en résultent dans les associations étudiantes et d'autres facteurs ${ }^{7}$. Dans tous ces cas, la nouveauté n'en est pas moins singulière, quant à sa capacité de se vêtir d'ancienneté.

L'utilisation de matériaux anciens pour construire à des fins entièrement originales des traditions inventées d'un type original paraît plus intéressante de notre point de vue. Un large éventail de tels matériaux s'est accumulé dans le passé de chaque société, et un langage élaboré de pratique et de communication symbolique est toujours disponible. Parfois, des traditions nouvelles ont pu être clairement greffées sur des anciennes, parfois elles ont pu être conçues par emprunt dans l'entrepôt bien fourni du rituel officiel, de l'exhortation symbolique et morale - la religion et le faste princier, le folklore et la francmaçonnerie (cette dernière étant elle-même une tradition inventée récente d'une grande force symbolique). Il en est ainsi du développement du nationalisme suisse, concomitant de la formation de l'État fédéral moderne au XIX ${ }^{\mathrm{e}}$ siècle, brillamment étudié par Rudolph Braun ${ }^{8}$ qui a l'avantage d'être formé dans une discipline ("Volkskunde ») qui se prête ellemême à de telles études, et dans un pays où la modernisation n'a pas été retardée par l'association avec les abus nazis. Il existe des pratiques coutumières traditionnelles chants populaires, lutte, adresse au tir - qui ont été modifiées, ritualisées et institutionnalisées pour répondre aux nouvelles finalités nationales. Des chants populaires traditionnels ont été remplacés par de nouvelles chansons écrites dans le même idiome, souvent composés par des instituteurs, et transférés dans un répertoire choral dont le contenu était patriotique-progressiste ( Nation, Nation, wie voll klingt der $\left.T^{T} n^{9} »\right)$, bien qu'il exprime aussi rituellement des éléments puissants de l'hymnologie religieuse. La formation de ces nouveaux répertoires, spécialement pour les écoles, mériterait d'être mieux étudiée. Les statuts du festival fédéral de la chanson - ne nous rappelle-t-il pas le eisteddfodau ${ }^{10}$ ? - déclarent que son objet consiste dans «le développement et l'amélioration du chant au sein du peuple, le réveil de sentiments plus élevés pour Dieu, la Liberté et le Pays, l'union et la fraternisation des amis de l'Art et de la Patrie " (le mot «amélioration» introduit une note caractéristique de l'idéologie du progrès du xix ${ }^{e}$ siècle).

Un puissant complexe rituel s'est mis en place à cette occasion: tentes de fête, échafaudages pour le déploiement des drapeaux, temples pour les offrandes, processions, sonnerie de cloches, saluts au canon, spectacles, délégations du gouvernement en l'honneur de la fête, dîners, toasts et discours. D'anciens matériaux ont été adaptés pour cela : «La répercussion des formes baroques de la célébration, de l'étalage et de la pompe 
est facilement reconnaissable dans cette nouvelle architecture de la fête. Et de même que, dans la célébration baroque, l'État et l'Église se fondent à un niveau supérieur, un alliage d'éléments religieux et patriotiques émerge de ces nouvelles formes de chant choral, de chasse et de gymnastique ${ }^{11}$.»

On ne peut discuter ici jusqu'à quel point les nouvelles traditions peuvent ainsi utiliser d'anciens matériaux, être obligées d'inventer de nouveaux langages et procédés ou d'étendre l'ancien vocabulaire symbolique au-delà de ses limites établies. Il est clair que beaucoup d'institutions politiques, de mouvements idéologiques et de groupes - pas seulement dans le cas du nationalisme - étaient tellement sans précédent que même la continuité historique a dû être inventée, en créant par exemple un passé ancien au-delà de la continuité historique effective, soit par semi-fiction (Boadicea, Vercingétorix, Hermann le Chérusque), soit par contrefaçon (Ossian, les manuscrits médiévaux tchèques). Il est tout aussi clair que des symboles et des procédés entièrement nouveaux ont émergé en tant qu'éléments des mouvements et des états nationaux, comme l'hymne national (l'hymne britannique, en 1740 , semble être le premier), le drapeau national (très largement une variation autour du drapeau tricolore révolutionnaire français, élaboré en 1790-1794), ou la personnification de la «nation" par un symbole ou une image, soit officielle comme pour Marianne et Germanie, soit non officielle, comme le dessin stéréotypé de John Bull, le maigre yankee Oncle Sam et " Michel le Germain ».

Par ailleurs, nous ne pouvons négliger la rupture dans la continuité qui peut parfois être visible même dans les topoi traditionnels d'une antiquité authentique. Si nous suivons Lloyd $^{12}$, la création de chants folkloriques anglais de Noël a cessé au XVII ${ }^{e}$ siècle; ils ont été remplacés par des cantiques venant de recueils de chants du type Watts-Wesley, quoiqu'on puisse observer une version populaire de ceux-ci dans des religions largement rurales comme le méthodisme primitif. Toutefois, les chorals sont les premiers types de chants folkloriques qui ont été relancés par les collecteurs bourgeois, pour prendre place "dans le nouveau cadre d'églises, de confréries et d'institutions féminines ", et, de là, s'étendre à un nouvel ensemble populaire urbain, " par les chanteurs de coin de rue ou par le garçon à la voix rauque chantant sur le pas des portes dans le vieil espoir d'une récompense ». En ce sens, «Dieu vous donne la joie, messieurs!» n'est pas ancien mais nouveau. Une telle rupture est également visible dans les mouvements qui se décrivent eux-mêmes comme "traditionalistes ", et qui séduisent des groupes qui sont considérés par tous comme les dépositaires de la continuité historique et de la tradition, tels que les paysans ${ }^{13}$. En effet, l'apparition même de mouvements de défense ou de renaissance des traditions, «traditionalistes » ou autres, indique une telle rupture. Communs parmi les intellectuels depuis les Romantiques, de tels mouvements ne peuvent jamais développer ou même préserver un passé vivant (sauf, comme on peut l'imaginer, en établissant des sanctuaires humains naturels pour conserver des coins de vie archaïque), mais ils sont condamnés à devenir une «tradition inventée ». D'un autre côté, la force et l'adaptabilité des traditions authentiques ne sont pas à confondre avec « l'invention de la tradition ». Là où les vieilles méthodes sont vivantes, les traditions n'ont besoin d'être ni renouvelées ni inventées.

18 Pourtant, on peut supposer que là où elles sont inventées, c'est souvent non parce que les vieux modèles ne sont plus valables ou viables, mais parce que délibérément ils ne sont plus utilisés ou adaptés. Ainsi, au $\mathrm{XIX}^{\mathrm{e}}$ siècle, en prenant position elle-même consciemment contre la tradition et pour l'innovation radicale, l'idéologie libérale du changement social a systématiquement échoué à établir des liens sociaux et d'autorité 
considérés comme allant de soi dans les sociétés antérieures; elle a ainsi créé des vides qui auraient pu être comblés par des pratiques inventées. Le succès des patrons conservateurs du Lancashire au XIX ${ }^{\mathrm{e}}$ siècle (différents à ce titre des patrons libéraux) dans la réutilisation, à leur avantage, de ces liens anciens montre qu'ils étaient toujours disponibles, même dans l'environnement sans précédent de la ville industrielle ${ }^{14}$. L'inadaptation à long terme des modèles préindustriels à une société transformée jusqu'à un certain point, ne fait pas de doute, mais elle ne doit pas être confondue avec les problèmes issus du rejet à court terme de vieux modèles par ceux qui les voyaient comme des obstacles au progrès, ou, pire encore, comme leurs adversaires militants.

Cela n'a pas empêché les innovateurs d'engendrer leurs propres traditions inventées - les pratiques de la franc-maçonnerie en sont un bon exemple. Néanmoins, une hostilité générale à l'irrationalisme, à la superstition et aux pratiques coutumières qui rappelaient des temps obscurs, même si elles ne provenaient pas réellement de ceux-ci, faisait de ces innovateurs des croyants passionnés en la vérité des Lumières ; c'est le cas des libéraux, des socialistes et des communistes, insensibles aux traditions anciennes ou nouvelles. Les socialistes, comme nous le verrons plus loin, se retrouvèrent avec un Premier Mai annuel sans presque savoir comment; les nationaux-socialistes exploitèrent de telles occasions avec zèle et raffinement liturgique, en manipulant consciemment les symboles ${ }^{15}$. L'ère libérale en Grande-Bretagne toléra, au mieux, de telles pratiques, tant que ni l'idéologie, ni l'efficacité économique ne furent en cause, parfois comme une concession faite à regret à l'irrationalisme des groupes sociaux inférieurs. Leur attitude devant les activités sociables et rituelles des Sociétés de Prévoyance était une combinaison d'hostilité («les dépenses inutiles » comme « les coûts des anniversaires, des processions, des orchestres, des insignes ", étaient légalement interdits) et de tolérance vis-à-vis d'événements comme les fêtes annuelles, sous prétexte qu'« on ne peut nier l'importance de cette attraction, en particulier par respect des habitants du pays ${ }^{16} »$. Mais un rationalisme individualiste rigoureux ne dominait pas seulement comme calcul économique, mais comme idéal social. Le chapitre $7^{17}$ explore ce qui se passa dans la période où ses limites s'imposèrent de plus en plus.

Nous pouvons conclure ces notes introductives par quelques observations générales sur les traditions inventées depuis la révolution industrielle.

21 Elles semblent appartenir à trois types qui se recoupent : a) celles qui établissent ou symbolisent la cohésion sociale ou l'appartenance à des groupes, des communautés réelles ou artificielles ; b) celles qui établissent ou légitiment des institutions, des statuts ou des relations d'autorité; c) celles dont le but principal était la socialisation, l'inculcation des croyances, des systèmes de valeur et des codes de conduite. Alors que les traditions de types b) et c) étaient certainement imaginées de toutes pièces (comme celles qui symbolisent la soumission à l'autorité dans l'Inde Britannique), nous pouvons provisoirement supposer que le type a) était courant, les autres fonctions étant perçues comme émanant implicitement ou comme découlant d'un sentiment d'identification à une «communauté » et/ou aux institutions qui la représentent, l'expriment ou la symbolisent en tant que « nation ».

Une difficulté tenait à ce que de telles entités sociales larges n'étaient pas simplement des Gemeinschaften, ou même des systèmes de rangs reconnus. La mobilité sociale, les conflits de classe et l'idéologie dominante engendraient des traditions qui combinaient la communauté à une inégalité marquée par des hiérarchies formelles (comme dans l'armée) difficilement applicables universellement. Cela n'affectait pas beaucoup les 
traditions de type $c$ ) puisque la socialisation générale inculquait les mêmes valeurs à chaque citoyen, membre de la nation et sujet de la Couronne, et que les spécificités fonctionnelles des formes de socialisation propres aux différents groupes sociaux (comme les écoliers de l'école publique en tant que distincts des autres) ne se faisaient pas habituellement obstacle les unes aux autres. Par ailleurs, dans la mesure où les traditions inventées réintroduisaient, si l'on peut dire, du «statut » dans un monde de «contrat ", des rapports hiérarchiques dans un monde d'égalité juridique, elles ne pouvaient pas le faire directement. Elles pouvaient être introduites en contrebande par consentement symbolique et formel à une organisation sociale qui était de fait inégale, comme pour le changement de style de la cérémonie britannique du couronnement ${ }^{18}$. Plus communément, elles pouvaient favoriser le sens collectif de la supériorité des élites particulièrement lorsque celles-ci devaient être recrutées parmi ceux qui ne la possèdent pas déjà par naissance ou par attribution - plutôt que d'inculquer un sentiment d'obéissance aux inférieurs. Certains étaient encouragés à se sentir plus égaux que les autres. Cela pouvait être fait en assimilant les élites aux groupes ou autorités prébourgeois dominants, soit sous la forme militariste/bureaucratique caractéristique de l'Allemagne (comme avec les corporations d'étudiants duellistes), soit sous la forme morale et non militarisée du modèle de la petite noblesse des "public schools» britanniques. Alternativement, peut-être, l'esprit de $\operatorname{corps}^{19}$, la confiance en soi et la domination des élites peuvent être développés par des «traditions " plus ésotériques marquant la cohésion d'un mandarinat officiel supérieur (comme en France ou parmi les blancs dans les colonies).

En admettant que les traditions inventées "communautaristes" représentent le type élémentaire, leur nature reste à étudier. L'anthropologie peut aider à élucider les différences, s'il y en a, entre les pratiques traditionnelles inventées et les pratiques anciennes. Ici, nous pouvons simplement noter ceci : alors que les rites de passage sont normalement inscrits dans les traditions de groupes particuliers (initiation, promotion, retraite, mort), ce n'était pas habituellement le cas de celles qui furent conçues pour des pseudo-communautés très englobantes (nations, pays), probablement parce que ces traditions affirmaient leur caractère immuable et éternel - du moins depuis la fondation de la communauté. Cependant, aussi bien les nouveaux régimes politiques que les mouvements innovateurs pouvaient s'efforcer de rechercher leurs propres équivalents par rapport aux rites traditionnels de passage associés à la religion (mariage civil, funérailles).

On peut observer une nette différence entre les pratiques anciennes et les pratiques inventées. Les premières étaient des pratiques sociales spécifiques, solidement établies, les suivantes tendaient à être assez indéterminées et vagues quant à la nature des valeurs, des droits et des obligations qu'elles inculquaient pour appartenir au groupe: " patriotisme », «loyauté », « devoir », " jouer le jeu», «l'esprit d'école», et ainsi de suite. Mais, si le contenu du patriotisme britannique ou de «l'américanisme» était particulièrement mal défini, bien que régulièrement précisé dans les discours associés aux occasions rituelles, les pratiques qui le symbolisaient étaient en fait obligatoires comme se lever pour chanter l'hymne national, ou le rituel du drapeau dans les écoles américaines. L'invention de signes d'adhésion au club, chargés symboliquement et émotionnellement, semble avoir été l'élément crucial, bien plus que les statuts et les objets du club. Leur signification réside précisément dans leur universalité indéfinie : «Le drapeau national, l'hymne national et l'emblème national sont les trois symboles par 
lesquels un pays indépendant proclame son identité et sa souveraineté, et en tant que tels ils inspirent respect immédiat et loyauté. En eux-mêmes, ils reflètent tout l'arrière-plan, l'esprit et la culture d'une nation ${ }^{20}$."

En ce sens, comme le note un observateur de 1880 , «c'est pour nous maintenant que soldats et policiers portent des insignes "; il ne pouvait prévoir leur renouveau comme accessoires pour de simples citoyens à l'époque des mouvements de masse qui en était à ses débuts ${ }^{21}$.

La seconde observation est qu'il semble clair qu'en dépit de beaucoup d'invention, les nouvelles traditions n'ont rempli qu'une petite partie de l'espace laissé par le déclin conjoint des anciennes traditions et des coutumes, comme on pouvait s'y attendre dans des sociétés où le passé devenait de moins en moins pertinent comme modèle ou exemple pour la plupart des formes du comportement humain. Dans la vie privée de la plupart des individus, et dans les vies autonomes de petits sous-groupes culturels, même les traditions inventées $d u \mathrm{XIX}^{\mathrm{e}}$ et $\mathrm{du} \mathrm{Xx}^{\mathrm{e}}$ siècle occupaient ou occupent une place beaucoup plus petite que celle qu'occupaient les anciennes traditions, par exemple, dans les sociétés agraires ${ }^{22}$. "Ce qui se fait » structure beaucoup moins le quotidien, les saisons et les cycles de vie des femmes et des hommes de l'Occident du $\mathrm{xx}^{\mathrm{e}}$ siècle, que chez leurs ancêtres, et beaucoup moins que les contraintes externes de l'économie, de la technologie, de l'organisation de l'État bureaucratique, des décisions politiques et d'autres forces qui ne s'appuient pas sur la tradition, ou ne la développent pas comme nous l'entendons.

27 Cette généralisation ne s'applique pas toutefois au champ de ce qui peut être appelé la vie publique des citoyens (celle-ci incluant en partie les formes publiques de socialisation, comme l'école, considérées comme distinctes des formes privées telles que les mass media). Il n'y a aucun signe réel d'affaiblissement des pratiques néo-traditionnelles associées aussi bien à la masse des individus dans le service public (les forces armées, le droit, voire les fonctionnaires) qu'aux pratiques liées à l'appartenance des citoyens à un État. En effet, la plupart des occasions où les individus prennent conscience de la citoyenneté en tant que telle, demeurent associées à des symboles et à des pratiques semi-rituelles (par exemple, les élections), qui sont pour la plupart historiquement nouveaux et largement inventés: drapeaux, images, cérémonies et musique. Dans la mesure où les traditions inventées de l'époque, depuis les révolutions industrielle et française, ont comblé une lacune permanente - ce qu'elles ont fait en tout cas jusqu'à aujourd'hui - elles semblent appartenir à ce champ.

On peut finalement se demander pourquoi les historiens doivent consacrer leur attention à de tels phénomènes. La question est en un certain sens inutile, depuis qu'un nombre croissant d'entre eux le font, comme en témoignent les textes de cet ouvrage et les références citées. Aussi, vaut-il mieux la reformuler ainsi : quel bénéfice les historiens peuvent-ils tirer de l'étude de l'invention de la tradition?

Nous pouvons d'abord et principalement suggérer que ces phénomènes sont des symptômes importants - et ainsi des indicateurs - de problèmes qui ne peuvent être reconnus autrement, et de développements qui seraient difficiles à identifier et à dater d'une autre façon. Ce sont des preuves. La transformation du nationalisme allemand, du vieux modèle libéral au nouveau modèle impérialiste-expansionniste, spécialement dans les années 1890, est mieux illustrée par le remplacement rapide des anciennes couleurs noir-rouge-or par les nouvelles couleurs noir-blanc-rouge, au sein du mouvement 
gymnique allemand, que par les déclarations officielles des autorités ou des porte-parole des organisations. L'histoire de la finale de la Coupe britannique nous dit bien plus sur le développement d'une culture ouvrière urbaine que ne le feraient des données et des sources plus conventionnelles. Ainsi, l'étude des traditions inventées ne peut être séparée d'une étude plus générale de l'histoire de la société : on ne peut espérer aller au-delà de la simple découverte de telles pratiques, si celle-ci n'est pas intégrée dans une étude plus globale.

Deuxièmement, cette étude projette une lumière considérable sur la relation de l'homme au passé et par là même sur l'objet et le métier propre aux historiens. Car toutes les traditions inventées utilisent, dans la mesure du possible, l'histoire comme source de légitimation de l'action et comme ciment de la cohésion du groupe. Fréquemment, elles deviennent le symbole réel des luttes, comme lors des conflits autour des monuments de Walther von der Vogelweide et de Dante dans le Sud Tyrol en 1889 et $1896^{23}$. Même les mouvements révolutionnaires ont souvent appuyé leurs innovations sur la référence à un "passé du peuple» (Saxons contre Normands, "nos ancêtres les Gaulois ${ }^{24}$ » contre les Francs, Spartacus), aux traditions de la révolution («Auch das deutsche Volk hat seine revolutionäre Tradition ${ }^{25}$ ", clamait Engels dans les premières lignes de sa Guerre des paysans en Allemagne ${ }^{26}$ ), et à leurs propres héros et martyrs. Labour in Irish History de James Connolly est un très bon exemple de l'union de ces thèmes. L'élément d'invention est ici parfaitement clair, depuis que l'histoire, qui est devenue une partie du fond de la connaissance ou de l'idéologie de la nation, de l'État ou des mouvements, n'est pas ce qui s'est conservé effectivement dans la mémoire populaire, mais ce qui a été sélectionné, écrit, peint, popularisé et institutionnalisé par ceux dont c'est la fonction. Les historiens de la tradition orale ont souvent observé combien dans la mémoire actuelle des anciens, la grève générale de 1926 jouait un rôle plus modeste et moins dramatique que ne le pensaient, à l'avance, les enquêteurs ${ }^{27}$. La formation d'une image semblable de la Révolution française pendant et par la ${ }{ }^{\mathrm{e}}{ }^{\mathrm{e}}$ République a déjà été analysée ${ }^{28}$. Tous les historiens, quels que soient leurs objectifs, sont aussi engagés dans ce processus dès lors qu'ils contribuent, consciemment ou non, à la création, au démantèlement et à la reconstruction d'images du passé qui n'appartiennent pas seulement à l'univers de l'investigation spécialisée mais aussi à la sphère publique de l'homme en tant qu'être politique. Ils pourraient être également conscients de cette dimension de leur activité.

31 À cet égard, il faut distinguer un intérêt spécifique des traditions inventées, en tout cas pour les historiens de la période moderne et contemporaine. Elles sont hautement pertinentes pour cette innovation historique relativement récente qu'est la «nation », avec ses phénomènes associés : le nationalisme, l'État-Nation, les symboles nationaux, les histoires nationales et le reste. Tous ces phénomènes prennent appui sur des exercices d'ingénierie sociale qui sont souvent délibérés et toujours innovants, ne serait-ce que parce que la nouveauté historique implique l'innovation. Le nationalisme israélien et palestinien, ou les nations correspondantes sont nécessairement une nouveauté, quelles que soient les continuités historiques auxquelles se réfèrent les juifs ou les musulmans du Moyen-Orient, puisque le concept même d'états territoriaux qui est actuellement le type standard dans leur région, était à peine conçu il y a cent ans, et qu'il ne devint guère une perspective sérieuse avant la fin du premier conflit mondial. Les langues nationales standards qui doivent être apprises à l'école et écrites, sans parler de l'oral, plus largement que par une petite élite, sont en grande partie des constructions d'époques diverses, mais souvent brèves. Comme l'observe assez justement un historien français de 
la langue flamande, le flamand parlé en Belgique aujourd'hui n'est pas la langue que les mères et les grands-mères flamandes parlaient à leurs enfants : en bref, c'est une « langue maternelle " au sens métaphorique et non littéral du terme. Nous ne devons pas être trompés par un paradoxe curieux, mais compréhensible: les nations modernes, et tous leurs impedimenta, revendiquent généralement d'être à l'opposé de la nouveauté - à savoir enracinées dans une antiquité lointaine - et à l'opposé de toute construction - à savoir des communautés humaines "naturelles » au point de n'avoir besoin d'une autre définition que leur propre affirmation. Quelles que soient les continuités, historiques ou autres, inscrites dans le concept moderne de «France» et de "Français » - et que personne ne chercherait à nier - ces concepts authentiques comportent nécessairement eux-mêmes un élément construit ou "inventé ». Parce que beaucoup de ce qui fait subjectivement la "nation" moderne consiste en de telles constructions et se trouve associé aux symboles appropriés, en général assez récents, ou à un discours convenablement adapté (comme «l'histoire nationale »), le phénomène national ne peut être étudié de manière adéquate sans une attention particulière portée à « l'invention de la tradition ». Enfin, l'étude de l'invention de la tradition est interdisciplinaire. C'est un champ d'étude qui rassemble aussi bien les historiens, les anthropologues sociaux que toute une variété d'autres chercheurs en sciences humaines, et qui ne peut être convenablement poursuivi sans une telle collaboration. Ce livre rassemble pour l'essentiel des contributions d'historiens. Il faut espérer que d'autres le jugeront également utile.

\section{NOTES}

1. Introduction au recueil d'études publié sous la direction de E. Hobsbawm \& T. Ranger, The Invention of Tradition, Cambridge, Cambridge University Press, 1983, p. 1-14. Nous remercions E. Hobsbawm et Cambridge University Press de nous avoir autorisés à en publier cette traduction. [NdlR.]

2. Parmi les approches critiques, A. D. Smith, «The Nation : Invented, Imagined, Reconstructed ", Millennium. Journal of International Studies, XX, 1991, p. 353-359. [NdlR.]

3. Par exemple, T. Ranger, " The Invention of Tradition Revisited. The Case of Colonial Africa ", in T. Ranger \& M. Vaughan, eds, Legitimacy and the State in Twentieth-Century Africa. Essays in Honour of A. H. M. Kirk-Green, Londres, Macmillan, 1993, p. 62-112. [NdlR.]

4. D. Cannadine, "The Context, Performance and Meaning of Ritual. The British Monarchy and the "Invention of Tradition", c. 1820-1977 ", in The Invention of Tradition, op. cit., p. 101-164.

5. Cf. G. Tihon, "Les religieuses en Belgique du $x I^{e}{ }^{e}$ au $x^{e}$ siècle: approche statistique ", Belgisch Tijdschrift V. Nieuwste Geschiedenis / Revue belge d'Histoire contemporaine, VII, 1976, p. 1-54.

6. K. Bahnson, Akademische Auszüge aus deutschen Universitäts un Hochschulorten, Sarrebrück, 1973.

7. Dix-sept exodes de ce type sont enregistrés au XVIII ${ }^{\mathrm{e}}$ siècle, cinquante entre 1800 et 1848 , mais seulement six de 1848 à 1973.

8. R. Braun, Socialer und kultureller Wandel in einem ländischen Industriegebiet im 19. und 20. Jahrhundert, Erlenbach-Zürich, 1965, chap. 6.

9. En allemand dans le texte. « Nation, Nation, que ce son sonne bien ».

10. Mot gallois désignant un concours de musique et de poésie [N.d.T.]. 
11. R. Braun, op. cit., p. 336-337.

12. A. L. Lloyd, Folk Song in England [1967], Londres, Lawrence and Wishart, 1969, p. 134-138.

13. Ceci doit être distingué de la renaissance de la tradition à des fins qui démontraient en fait son déclin. «La reprise par les fermiers (vers 1900) de leurs vieux habits régionaux, des danses folkloriques et autres rituels similaires pour des occasions festives, n'était une caractéristique $n i$ bourgeoise $n i$ traditionaliste. En apparence, cela peut être perçu comme une nostalgie de la culture du bon vieux temps qui disparaissait si rapidement, mais en réalité, c'était une démonstration d'identité de classe par laquelle les fermiers prospères pouvaient prendre leur distance, horizontalement, des citadins et, verticalement, des paysans, des artisans et des ouvriers ». P. O. Christiansen, « Peasant in the Danish Countryside », Ethnologia Scandinavica, VIII, 1978, p. 128. Cf. aussi G. Lewis, «The Peasantry, Rural Change and Conservative Agrarianism. Lower Austria at the Turn of the Century », Past and Present, 81, 1978, p. 119-143.

14. P. Joyce, "The Factory Politics of Lancashire in the Later Nineteenth Century ", Historical Journal, XVIII, 1965, p. 525-553.

15. H. Hartwig, «Plaketten zum 1. Mai 1934-39», Asthetik und Kommunikation, VII, 26, 1976, p. 56-59.

16. P. H. J.H. Gosden, The Friendly Societies in England, 1815-1875, Manchester, Manchester University Press, 1961, p. 123,119.

17. E. Hobsbawm, « Mass-Producing Traditions : Europe, 1870-1914», in The Invention of Tradition, op. cit., p. 263-307.

18. J. E. C. Bodley, The Coronation of Edward the Seventh. A Chapter of European and Impérial History, Londres, Methuen, 1903, p. 201,204.

19. En français dans le texte.

20. Commentaire officiel du gouvernement de l'Inde cité par R. Firth, Symbols, Public and Private, Londres, Cornell University Press, 1973, p. 341.

21. F. Marshall, Curiosities of Ceremonials. Tittes, Decorations and Forms of International Vanities, Londres, 1880, p. 20.

22. Sans mentionner la transformation des rituels durables et des signes d'uniformité et de cohésion en modes qui changent rapidement - dans le costume, le langage, la pratique sociale, etc., comme dans la culture des jeunes des pays industrialisés.

23. J. W. Cole \& E. Wolf, The Hidden Frontier. Ecology and Ethnicity in an Alpine Valley, New York, Academic Press, 1974, p. 55.

24. En français dans le texte [N.d.T.].

25. En allemand dans le texte : « Le peuple allemand aussi a sa tradition révolutionnaire » [N.d.T.].

26. Pour la popularité, dans les bibliothèques des ouvriers allemands, des livres d'histoire militante, sur ces sujets, ou sur d'autres, cf. H.-J. Steinberg, Sozialismus und deutsche Sozialdemokratie. Zur Ideologie der Partei vor dem ersten Weltkrieg, Hanovre, 1967, p. 131-133.

27. II y a de solides raisons pour expliquer que les participants de la base ne voient pas d'ordinaire comme les dominants ou les historiens les événements historiques qu'ils vivent. On pourrait appeler cela, d'après le héros de La Chartreuse de Parme de Stendhal, le «syndrome de Fabrice ».

28. Par exemple A. Gérard, La Révolution française. Mythes et interprétations, 1789-1970, Paris, Flammarion, 1970. 


\section{RÉSUMÉS}

Les traditions inventées, qui dérivent essentiellement d'un procès de formalisation et de ritualisation, n'ont pas été étudiées par les historiens de façon satisfaisante. Distinctes des coutumes, qui définissent les sociétés traditionnelles, elles sont étroitement liées aux transformations rapides des sociétés modernes. Elles contribuent à l'établissement de la cohésion sociale, à la légitimation des institutions ou de l'autorité mais aussi à la socialisation des croyances ou des systèmes de valeurs. En utilisant, jusqu'à un certain point, des matériaux issus du passé, elles jouent un rôle prééminent dans la constitution de cette innovation relativement récente qu'est la nation.

The process of inventing traditions which is essentially a process of formalization and ritualization has not been adequately studied by historians. As distinct from customs which define traditional societies, invented traditions are closely linked to the rapid transformations of modern societies. They contribute to the establishment of social cohesion, to the legitimation of institutions or authority as well as to the socialization of beliefs and value systems. Utilizing to some extent materials drawn from the past, they play a prominent role in the making of this relatively recent innovation that is the nation 